\title{
Open Reduction and Internal Fixation Using Double Plating with Biological and Artificial Bone Grafting of Aseptic Non-unions of the Distal Humerus: Clinical Results
}

\author{
Giuseppe Rollo ${ }^{1}$, Giovanni Vicenti ${ }^{2}$, Roberto Rotini ${ }^{3}$, Ante Prkic ${ }^{4}$, Denise Eygendaal ${ }^{5}$, Luigi Meccariello ${ }^{6}$
}

\begin{abstract}
Aim: Intra-articular non-union of fractures is an uncommon but complex problem because in general, it is characterised by marked instability, pain, strength loss and significant functional limitation. The aim of this study is to report our prospective medium-term outcomes of the treatment of intra-articular, distal humeral aseptic non-unions using open reduction and internal fixation, augmented with artificial bone.

Materials and methods: A retrospective case series of 16 patients with intra-articular, aseptic non-unions of the distal humerus was analysed for range of motion, pain, Mayo Elbow Performance Scores (MEPS) and Oxford Elbow Scores (OES) after 12 months. Mean age was 44 years (range, 18-84 years) and mean total follow-up was 43 months (range, 24-62 months).

Results: All subjective and objective scores were significantly higher 12 months after treatment with internal fixation and artificial bone augmentation; the mean improvement on the MEPS was 18 points and 17 points on the OES. All patients returned to work, most without limitations. Autografts had worse outcomes compared to allografts regarding post-operative pain and time to return to work. No adverse events related to the artificial bone augmentation were seen and all fractures consolidated.

Conclusion: The use of two locking plates and bone graft augmentation with autografts or allografts with artificial bone grafts is a successful treatment of intra-articular distal humeral non-unions after hardware failure or biological limitations.

Clinical significance: The use of artificial bone in the treatment of septic non-unions of the upper limb is safe. When no autograft is possible because of concurrent morbidity, it can be used alone or combined with an allograft to reconstruct the affected bone without leading to extra morbidity or complications.

Keywords: Artificial bone grafting, Aseptic non-union, Distal humerus, Patient-reported outcomes, Reconstruction, Revision surgery.

Strategies in Trauma and Limb Reconstruction (2021): 10.5005/jp-journals-10080-1533
\end{abstract}

\section{INTRODUCTION}

Most often, a distal humerus non-union is located at the supracondylar level with the articular fragments having healed in a near-anatomic position. Intra-articular non-union of fractures is an uncommon but complex problem because in general, it is characterised by marked instability, pain, strength loss and significant functional limitation. ${ }^{1,2}$ The reported incidence of non-union after surgical treatment of distal humerus fractures ranges between 2 and $10 \% .^{3,4}$ Since the distal humerus is a unique anatomical structure that has to withstand forces in multiple planes and directions, treatment becomes different from that of other long bone fractures. ${ }^{3}$ Anatomical restoration of length, alignment, rotation and the distal humeral cartilage maintains the optimal stabilising effects of the elbow and forearm muscles and therefore leads to better function and higher patient-reported outcomes. ${ }^{3,5}$ Complex fractures, poor bone quality, soft tissue lesions and patients' comorbidities, particularly if associated with incorrect or inadequate internal fixation, favour complications. Open reduction and internal fixation (ORIF) with plates and screws is the treatment of choice in active patients. ${ }^{1-3,6,7}$ If surgery is indicated, it must restore function in a long-lasting way as this is necessary to achieve painless bone union and to restore an acceptable range of motion (ROM). ${ }^{7}$ Bone (allo)graft addition to the non-union focus is used more commonly in the upper limb during reconstructive surgery. ${ }^{8-10}$ After a septic non-union was ruled out using history, physical examination

\footnotetext{
1,6Department of Orthopedics and Traumatology, Vito Fazzi Hospital, Lecce, Italy

${ }^{2}$ Department of Basic Medical Sciences, Neuroscience and Sense Organs, School of Medicine, University of Bari "Aldo Moro"-AOU Policlinico Consorziale, Bari, Italy; Orthopaedic and Trauma Unit, Bari, Italy

${ }^{3}$ Shoulder and Elbow Unit, "Rizzoli" Orthopaedic Institute, Bologna, Italy

${ }^{4}$ Department of Orthopedic Surgery, Amphia Hospital, Breda, The Netherlands

${ }^{5}$ Department of Orthopedic Surgery, Amphia Hospital, Breda, The Netherlands; Department of Orthopedic Surgery, AUMC, Amsterdam, The Netherlands
}

Corresponding Author: Ante Prkic, Department of Orthopedic Surgery, Amphia Hospital, Breda, The Netherlands, Phone: +31648340436; e-mail: aprkic@live.nl

How to cite this article: Rollo G, Vicenti G, Rotini R, et al. Open Reduction and Internal Fixation Using Double Plating with Biological and Artificial Bone Grafting of Aseptic Non-unions of the Distal Humerus: Clinical Results. Strategies Trauma Limb Reconstr 2021;16(3):144-151.

Source of support: Nil

Conflict of interest: None

() The Author(s). 2021 Open Access This article is distributed under the terms of the Creative Commons Attribution-Non Commercial-share alike license (https://creativecommons.org/licenses/by-nc-sa/4.0/) which permits unrestricted distribution, and non-commercial reproduction in any medium, provided you give appropriate credit to the original author(s) and the source, provide a link to the Creative Commons license, and indicate if changes were made. If you remix, transform, or build upon the material, you must distribute your contributions under the same license as original. The Creative Commons Public Domain Dedication waiver (http://creativecommons.org/publicdomain/zero/1.0/) applies to the data made available in this article, unless otherwise stated. 
and laboratory tests (C-reactive protein, leucocyte count), the patients were informed in a clear and comprehensive way about the possible surgical and conservative alternatives during their outpatient visit at our referral centre. When they consented for a surgical revision of the non-union, they were treated according to the ethical standards of the Helsinki Declaration and were invited to read, understand and sign the informed consent form.

The aim of this study is to report our prospective medium-term outcomes of the treatment of intra-articular, distal humeral aseptic non-union using ORIF with allograft bone grafting.

\section{Materials and Methods}

\section{Patient Selection}

From a total of 34 patients with intra-articular, distal humeral non-unions, we included 16 patients with aseptic non-unions in this retrospective case series after using the following exclusion criteria: previous distal humeral fractures, oncological patients, age under 18 years, bone metabolism disease, pre-existent elbow osteoarthritis, rheumatoid disease, acute or chronic infections and ASAMI non-union classification's type $A$ and $C .^{11,12}$ Intra-articular fracture was defined as a primarily intra-articular fracture pattern, in which the articular surface was not necessarily the most problematic non-union site. The institutional review board of our institution approved this study.

\section{Peri-surgical Care}

Surgery took place in a prone position with the arm on a radiolucent support, or a padded post as either gives maximum freedom of space to a posterior approach of the elbow joint. A triceps-on, transtricipital approach was tried first to preserve triceps function; when the view on the articular aspect was insufficient, an olecranon osteotomy was performed..$^{13}$ The ulnar nerve was always identified, released and protected during surgery. In the cases where the radial or median nerve were injured, the corresponding nerves were identified and neurolysed up to a level where the nerve seemed normal, but never transposed.

After exposure of the non-union focus, we removed the previous implant(s) and reamed the distal humeral shaft to remove fibrous tissue and to promote blood supply to the non-union site. Debridement of the fracture site was performed further until healthy, bleeding cortical bone was reached. This therefore resulted in shortening in some cases where the complete circumference of the distal humerus was affected, as the cortices of the proximal and distal side were opposed as much as possible with as much contact as possible in these cases.

After renewed reduction, fixation was performed with two plates (LCP ${ }^{\oplus}$, DePuy Synthes ${ }^{\mathrm{TM}}$, Oberdorf, Switzerland) in orthogonal or parallel configuration, depending on the surgeon's expertise in each specific case. Frozen, decellularised bone chips were put as an augmentation inside the humeral shaft and compressed until the canal and non-union/fracture site were filled. Only when patients denied the use of allografts, a fibular autograft was retrieved during the same surgery. Remaining cortical gaps were augmented using Putty ${ }^{\circledR}$ Biocollagen Crunch bone pasta (Biogen ${ }^{\circledR}$, Bioteck ${ }^{\top \mathrm{M}}$, Arcugnano, Vicenza, Italy), over the decellularised bone chips that were put inside the humeral shaft. At the end of the surgery, the result was inspected with fluoroscopy in three different views (antero-posterior, medial-lateral and false oblique) and dynamic tests of the elbow were performed to check for intra-articular screw placement. The triceps was re-attached with muscle side-to-side sutures using absorbable sutures, and the olecranon osteotomies were fixated using olecranon plates, screws or K-wire tension bending according to the surgeon's preference, with regard to the patients' characteristics.

After closure of the fascia and subcutaneous tissue, the skin was closed with metal staples. A resin semicircular cast was applied for the first 3 weeks. The cast ranged from the metacarpals to the humeral shaft, with the elbow flexed at $90^{\circ}$.

All patients underwent the same rehabilitation protocol (see Appendix 1, rehabilitation protocol). To study the bone healing on radiographs, we used the Non-Union Scoring System (NUSS) in retrospective mode (Table 1). ${ }^{14}$ Patients had a follow-up of 5 years, and after 1 year, the subjective and objective quality of life and the elbow function were measured by the Mayo Elbow Performance Score (MEPS), the subjective quality of life and the elbow function were measured by the Oxford Elbow Score (OES). Both questionnaires were set with 0 as 'worst possible outcome' and 100 as 'best possible outcome'. Bone union was measured using the radiographic union score during follow-up as described by Radiographic Union Score (RUS). ${ }^{15}$ Pain visual analogic scores (VAS) were collected the same day that the radiographs were taken. As the evaluation endpoint was set at 12 months after surgery, the patients were asked for satisfaction and return to work at that time.

\section{Statistical Analysis}

Descriptive statistics were used to summarise the characteristics of the study group and subgroups, including means and standard deviations of all continuous variables. The Student's $t$-test was used to compare continuous outcomes when normality was met; otherwise, the Mann-Whitney $U$ test was used. The Fisher exact test was used in groups smaller than 10 patients to compare categorical variables. The statistical significance was defined as $p<0.05$. We used Pearson's correlation coefficient $(r)$ to compare the predictive score of outcomes and quality of life. Statistical analyses were performed with SPSS v.15.0 (SPSS Inc., IBM, Chicago, Illinois, USA). Mean ages (and their standard deviations) of the patients were rounded at the closest year. The predictive score of outcomes and quality of life and their standard deviations were approximated at the first decimal while the second decimal was approximated by Pearson's correlation coefficient.

Cohen's kappa coefficient $(\kappa)$ is a statistic which measures inter-rater agreement for qualitative (categorical) items. With this parameter, we calculated the concordance between different qualitative values of the outcomes and the bone healing, the anatomical and biomechanical axes of the humerus from the radiological point of view, measured with the trochleocapitellar index. This parameter has an optimal value, as too much varus or valgus within the elbow joint does lead to worsened outcomes. ${ }^{5}$

\section{RESULTS}

Of the 16 included patients, the mean age was 44 years (range, 18-84 years) and mean follow-up was 43 months (range, 24-62 months). The injury pattern consisted of high-impact injuries in 11 cases, and most (9 patients) had a C2 fracture type when classified by the Arbeitsgemeinschaft für Osteosynthesefragen classification. The most used method of primary fixation was plate fixation (six parallel, six orthogonal, two single plates). Duration of non-union varied between 2 and 11 months, with four times fixation failure as the reason of non-union. Five patients had nerve damage, of which one had combined ulnar, median and radial nerve damage. 


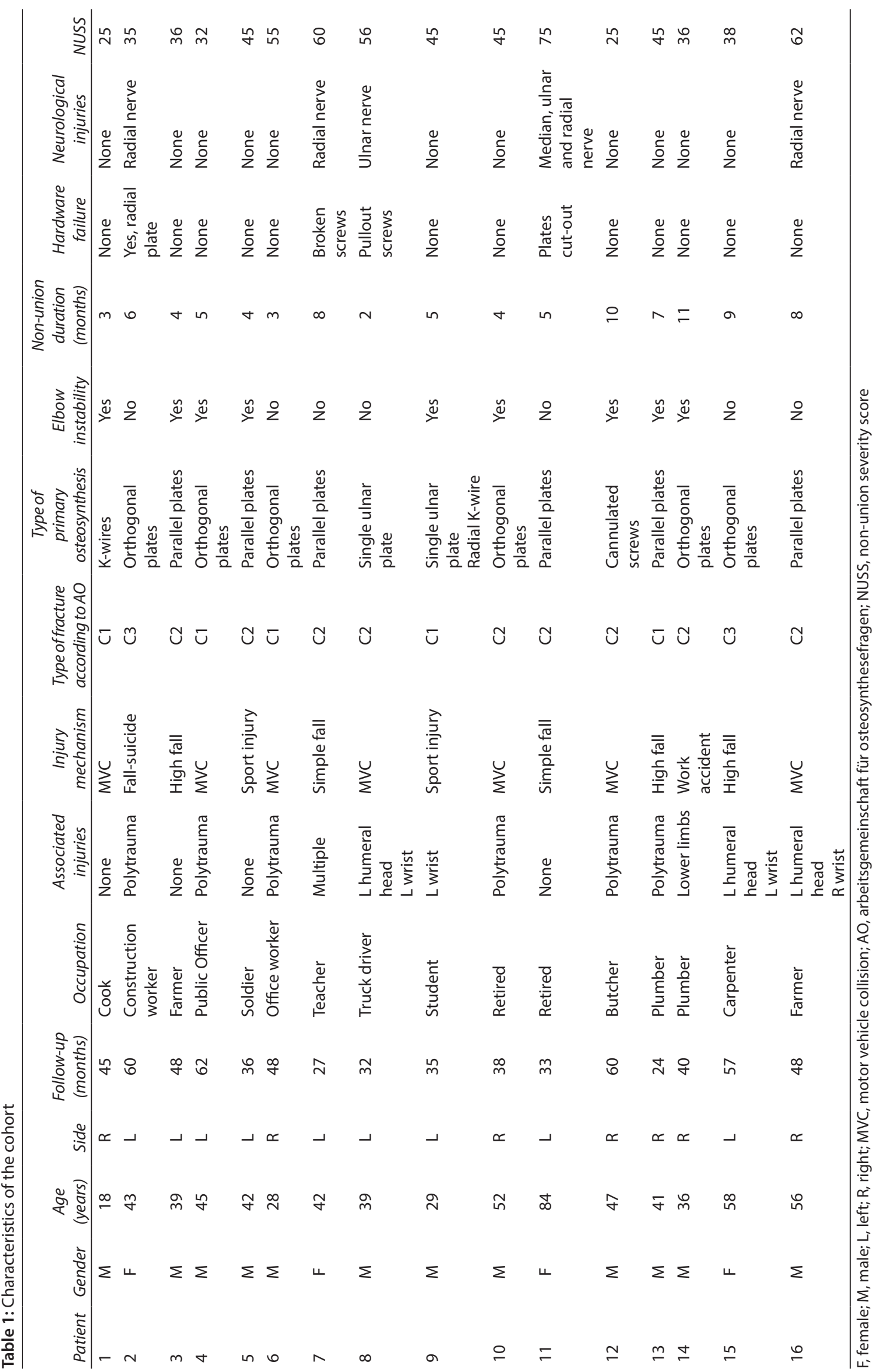




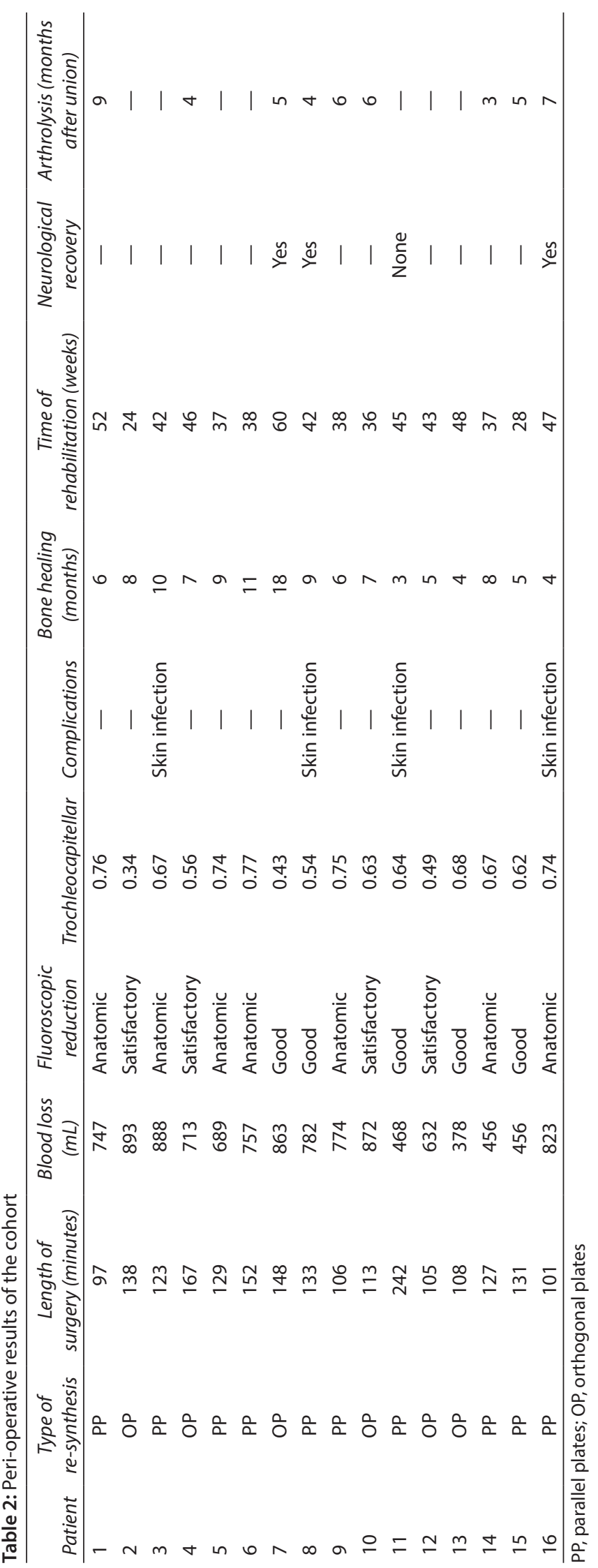

The mean NUSS score was 45 points ( $\pm 14 ; 25-75$ points). Further specific demographic pre-operative details are listed in Table 1.

The surgical data are summarised in Table 2 . Six patients received orthogonally placed plates and 10 patients parallel plates because of their fracture pattern. Mean trochleocapitellar index was 0.63 (standard deviation \pm 0.13 , range $0.34-0.77$ ). Four patients had a superficial wound infection successfully treated with antibiotics. All wounds healed within 3 weeks, and no new neurological deficits occurred. The neurological deficits that existed pre-operatively resolved over time, except for the patient with the combined ulnar, median and radial nerve injury. This patient was the only one not to return to their preoperative functional level; nine patients returned to their own work and six patients needed adaptations yet still returned to their own work. During follow-up, nine patients underwent an open arthrolysis because of capsular contractions. Of these, the mean improvement on the MEPS was 18 points and 17 points on the OES. One patient did not improve, as can be seen in Table 3.

The average time to full bone healing on radiographs was 30 weeks (range, 12-72 weeks). The average time to full functional rehabilitation was 42 weeks (range, 24-60 weeks). Average return to work was possible after 38 weeks (range, 24-60 weeks) for patients who received an allograft and after 50 weeks (range, 16-120 weeks) for patients who received an autograft ( $p=0.036)$. On the day of resuming work, the VAS pain scores showed a mean of 1.4 ( \pm 0.4 ; range $0-3$ points) in the allograft group and 2.8 ( \pm 0.8 ; range $0-4$ points) in the autograft group ( $p=0.045$ ). At this moment, the correlation between RUS and VAS scores showed a Pearson's $r=0.041$ in the allograft group and $r=0.059$ for the autograft group ( $p=0.039$ ).

After 1 year, all patient-reported outcomes were significantly higher than during the non-union period. The ROM also was higher with less pain. The average correlation between radiographic union and the clinical outcomes was $k=0.86( \pm 0.11)$. A patient's course from pre-operative work-up to follow-up is illustrated in Figure 1.

\section{Discussion}

This study showed good radiographic and clinical results after the use of augmentation in aseptic non-unions of intraarticular distal humeral fractures. As these fractures need optimal restoration of the cartilage contours for best functional outcomes and sufficient stability to achieve (radiographic) union, our treatment using plate fixation and additional bone grafting seems to offer good results. In most cases, primary fixation failed because of inadequate stability or biological circumstances. Compared to previous studies with comparable techniques at this centre concerning more proximal humeral aseptic non-unions, the radiographic and patient-reported outcomes are comparable..$^{9,10,16}$ Therefore, we believe the addition of bone grafts, either autografts, allografts or artificial, to debrided non-union sites and sufficient stability is an adequate treatment. Other authors describe similar techniques with the same philosophy and good outcomes. ${ }^{2,3}$

The difference in our series is the application of artificial bone graft, compared to allograft or autograft in the largest series published in the literature. ${ }^{2}$ As seen in the results, the group of patients who received an allograft performed better than those with an autograft; pain scores and time to return to work seem beneficial for the allograft, most logically because of less 
ORIF and Artificial Bone Grafting for Non-unions of the Distal Humerus

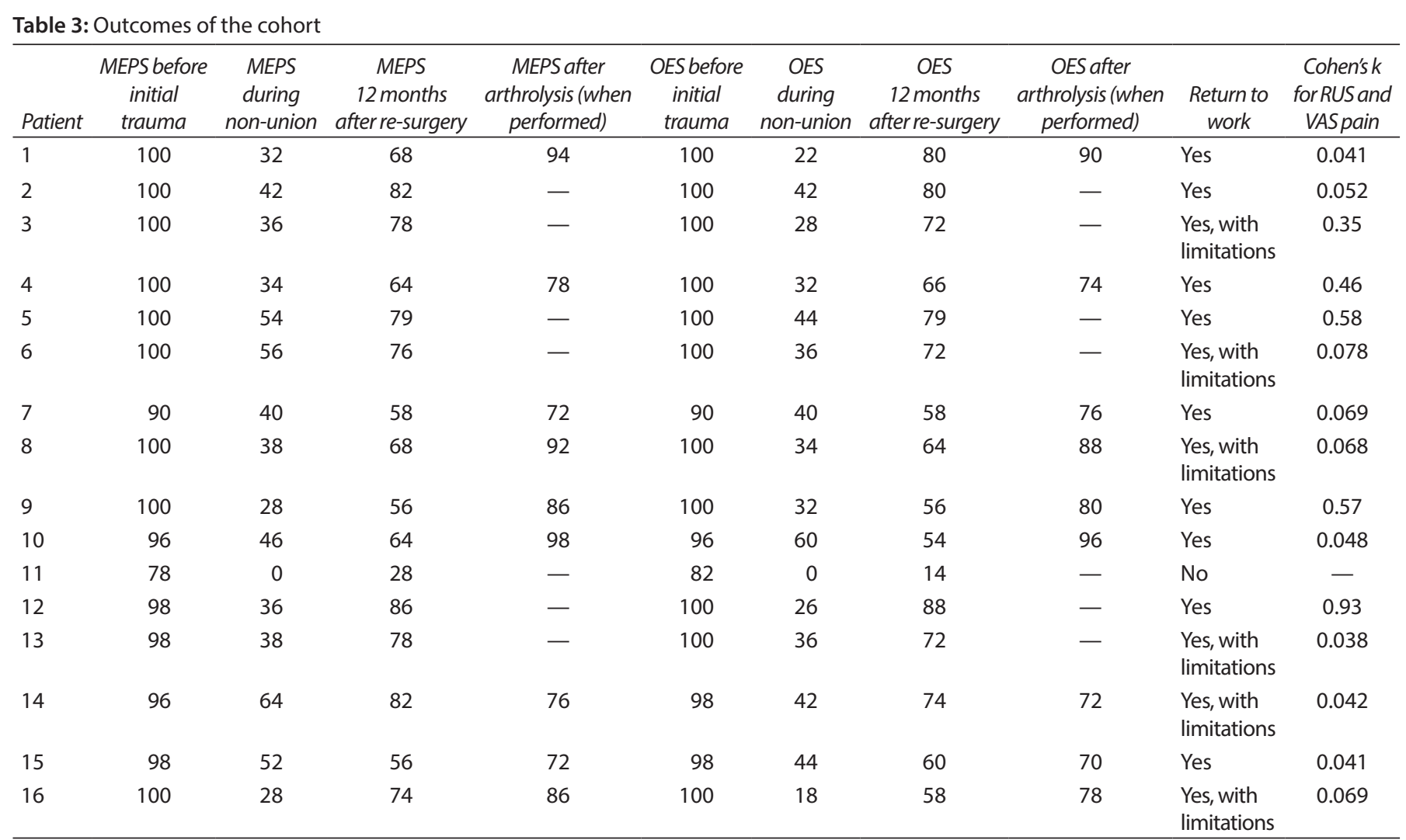

MEPS, Mayo elbow performance score; OES, Oxford elbow scale; RUS, radiographic union score; VAS, visual analogue scale

extra morbidity arising from autograft retrieval. The allograft has no extra patient morbidity in a patient group that mostly already has sustained a severe trauma. Artificial bone grafts may have the extra advantage that they are limitlessly available, have different options to choose from and do not require special storage conditions, such as refrigerators. However, their exact mechanism of work is still investigated. ${ }^{17,18}$ Moreover, the number of patients in our study is relatively low to draw well-funded conclusions. The superficial skin infection rate is high (25\%) yet these cases were managed well with antibiotic therapy only and no deep infections occurred. In contrast to the mentioned study, antibiotics were given to our patients prior to surgery instead of during surgery after sampling of tissues for lowgrade infections, and Augmentin treatment for 6 days after surgery was given on a regular basis after surgery. ${ }^{2}$

In this study, we have assessed return to work, which is an important outcome in this longstanding elbow pathology; the mean return to work was 38 weeks, which is more than the 90 -day period which is important in other orthopaedic-traumatic pathology. ${ }^{19}$ Nevertheless, all patients who worked returned to work, with more than half without limitations after a mean follow-up of 43 months. However, the longer-term outcomes have to be awaited, as elbows are prone to post-traumatic degeneration. ${ }^{20}$

The patients who underwent arthrolysis had a further, expected, increase of ROM after arthrolysis. As described by Donders et al., it is possible to perform during the same surgery when deemed necessary. ${ }^{2}$ In our view, it is difficult to determine pre- or perioperatively which patient will develop a stiff elbow; therefore, the capsular release was very limited in our series to prevent more extensive bleeding and surgery time. The result is that more than half of the patients needed a second surgery, which was successful in all but one patient who did not improve on the MEPS and OES; the other eight patients who underwent arthrolysis benefited greatly.

However, this case series is an uncontrolled retrospective case review in a relatively small group of patients with a specific condition. Therefore, this technique is not validated in large cohorts and the extra value of the artificial bone grafting over allografts of autografts was not studied. Moreover, because of the specific nature of the aseptic, intra-articular non-unions of the distal humerus, no clear guidance on bone resection or generalisable surgical steps can be stated; every case has its specific details that need to be addressed. On the other side, the surgical technique as used for our patients relies on the concepts of proper fixation, adequate soft tissue envelope and favourable biologic circumstances at the fracture site. Because of the lack of a control group with only debridement and renewed fixation without any (artificial) bone grafting, we cannot elaborate on the effect of the bone grafts.

Regarding the definition of non-union, the time between primary management of the fractures and time to the re-intervention with our technique was not 6 months for all cases. In four of nine cases performed within 6 months, there was either hardware failure or evident inadequate fixation, and the other five patients had malalignment without callus; therefore waiting for a longer period was not warranted and we considered this as a non-union as further spontaneous union with good results was not to be expected in these cases.

\section{Conclusion}

The use of two locking plates and bone graft augmentation with autografts or allografts with artificial bone grafts is a successful 


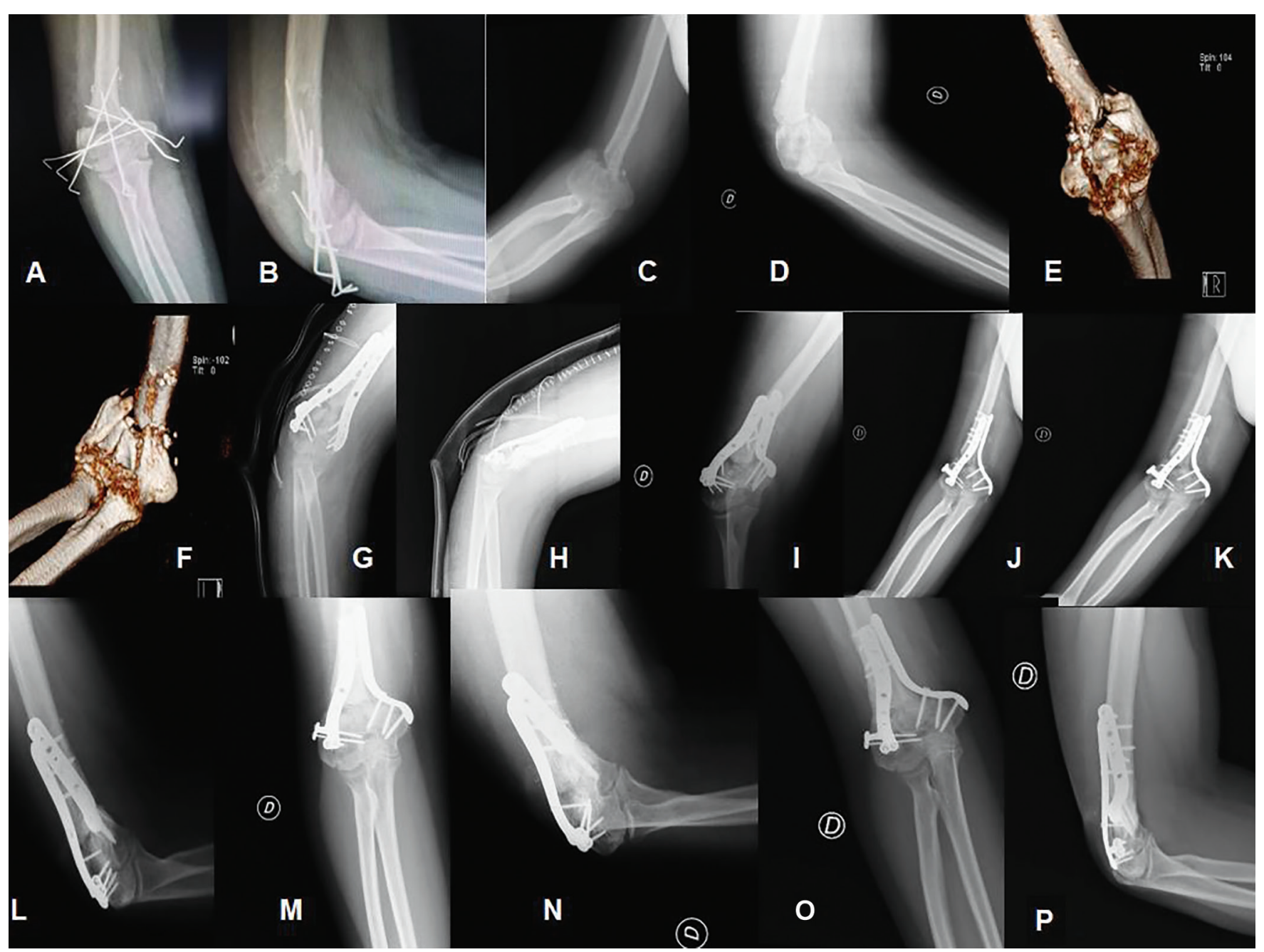

Figs 1 A to Q: (A and B): Initial situation at time of referral, 3 weeks after treatment elsewhere; (C and D): Situation after removal of K-wires at our outpatient clinic; (E and F): Three-dimensional views of the CT, made to assess comminution; ( $\mathrm{G}$ and $\mathrm{H})$ : First post-operative radiographs; (I and J): One month after surgery, without loss of reduction; (K and L): Two months after surgery, with progression of bone union; ( $M$ and $N)$ : Three months after surgery; ( $\mathrm{O}, \mathrm{P}$ and $\mathrm{Q})$ : Six months after surgery, with completed one healing

treatment of intra-articular distal humeral non-unions after hardware failure or biological limitations.

We did not encounter graft-related problems in our retrospective case series, yet a large proportion of patients needed an arthrolysis of the elbow because of secondary stiffness.

\section{Clinical Significance}

The use of artificial bone in the treatment of aseptic non-unions of the upper limb is safe. When no autograft is possible because of concurrent morbidity, it can be used alone or combined with an allograft to reconstruct the affected bone without leading to extra morbidity or complications.

\section{References}

1. Allende $C$, Allende BT. Post-traumatic distal humerus non-union: open reduction and internal fixation: long-term results. Int Orthop 2009;33(5):1289-1294. DOI: 10.1007/s00264-008-0650-8.

2. Donders JCE, Lorich DG, Helfet DL, et al. Surgical technique: treatment of distal humerus nonunions. HSS J 2017;13(3):282-291. DOI: 10.1007/ s11420-017-9551-y.
3. Helfet DL, Kloen $\mathrm{P}$, Anand $\mathrm{N}$, et al. Open reduction and internal fixation of delayed unions and nonunions of fractures of the distal part of the humerus. J Bone Jt Surg - Ser A 2003;85(1):33-40. DOI: 10.2106/00004623-200301000-00006.

4. Bégué T. Articular fractures of the distal humerus. Orthop Traumatol Surg Res 2014;100(1 Suppl.). DOI: 10.1016/j.otsr.2013.11.002.

5. Rollo G, Rotini R, Eygendaal D, et al. Effect of trochleocapitellar index on adult patient-reported outcomes after noncomminuted intraarticular distal humeral fractures. J Shoulder Elb Surg 2018;27(7): 1326-1332. DOI: 10.1016/j.jse.2018.02.073.

6. Simonis RB, Nuñez VA, Khaleel A. Use of the coventry infant hip screw in the treatment of nonunion of fractures of the distal humerus. J Bone Jt Surg - Ser B 2003;85(1):74-77. DOI: 10.1302/ 0301-620X.85B1.12953.

7. Pogliacomi F, Aliani D, Cavaciocchi M, et al. Total elbow arthroplasty in distal humeral nonunion: Clinical and radiographic evaluation after a minimum follow-up of three years. J Shoulder Elb Surg 2015;24(12):1998-2007. DOI: 10.1016/j.jse.2015.08.010.

8. Rollo G, Pichierri P, Marsilio A, et al. The challenge of nonunion after osteosynthesis of the clavicle: Is it a biomechanical or infection problem? Clin Cases Miner Bone Metab 2017;14(3):372-378. DOI: 10.11138/ccmbm/2017.14.3.372. 
9. Rollo G, Rotini R, Pichierri $P$, et al. Grafting and fixation of proximal humeral aseptic non union: a prospective case series. Clin Cases Miner Bone Metab 2017;14(3):298-304. DOI: 10.11138/ $\mathrm{ccmbm} / 2017.14 .3 .298$.

10. Rollo G, Prkić A, Pichierri P, et al. Plate-and-bone-strut fixation of distal third humeral shaft aseptic non-unions: a consecutive case series. J Clin Orthop Trauma 2019;10:S127-S132. DOI: 10.1016/j. jcot.2019.05.004.

11. Paley D, Catagni MA, Argnani F, et al. llizarov treatment of tibial nonunions with bone loss. Clin Orthop Relat Res 1989;(241):146-165. PMID: 2924458.

12. Yin $\mathrm{P}, \mathrm{Zhang} \mathrm{L}$, Li T, et al. Infected nonunion of tibia and femur treated by bone transport. J Orthop Surg Res 2015;10(1):49. DOI: 10.1186/ s13018-015-0189-5.

13. Morrey BF, Sanchez-Sotelo J. Approaches for elbow arthroplasty: how to handle the triceps. J Shoulder Elbow Surg 2011;20(2 Suppl.): S90-S96. DOI: 10.1016/j.jse.2010.12.004.

14. Calori GM, Colombo M, Mazza EL, et al. Validation of the Non-Union Scoring System in 300 long bone non-unions. Injury 2014;45(S6): S93-S97. DOI: 10.1016/j.injury.2014.10.030.
15. Maiettini D, Bisaccia M, Caraffa A, et al. Feasibility and value of Radiographic Union Score Hip fracture after treatment with intramedullary nail of stable hip fractures. Acta Inform Med 2016;24(6):394-396. DOI: 10.5455/aim.2016.24.394-396.

16. Rollo G, Prkic A, Bisaccia $M$, et al. Grafting and fixation after aseptic non-union of the humeral shaft: a case series. J Clin Orthop Trauma 2020;11:S51-S55. DOI: 10.1016/j.jcot.2019.08.020.

17. Bose $S$, Roy M, Bandyopadhyay A. Recent advances in bone tissue engineering scaffolds. Trends Biotechnol 2012;30(10):546-554. DOI: 10.1016/j.tibtech.2012.07.005.

18. Mariani E, Lisignoli G, Borzì RM, et al. Biomaterials: foreign bodies or tuners for the immune response? Int J Mol Sci 2019;20(3):636. DOI: 10.3390/ijms20030636.

19. Murgatroyd DF, Harris IA, Tran $Y$, et al. Predictors of return to work following motor vehicle related orthopaedic trauma. BMC Musculoskelet Disord 2016;17(1):171. DOI: 10.1186/s12891-0161019-6.

20. Macken AA, Prkic A, Kodde IF, et al. Global trends in indications for total elbow arthroplasty: a systematic review of national registries. EFORT Open Rev 2020;5(4):215-220. DOI: 10.1302/2058-5241.5.190036. 


\section{Appendix I}

\section{Rehabilitation Protocol}

The purpose of our protocol is to provide the clinician with an orientation of the post-operative course of rehabilitation, to rationalise and to have the whole patient population conform to a single physiotherapy program.

\section{Week 1-3}

During the first three post -operative weeks the patients wore a resin cast from the humerus to the metacarpals with the elbow flexed in $90^{\circ}$.

\section{Week 4-6}

After the first three weeks the patient received a Hinged Elbow Brace.

- Week 4: Full elbow flexion, up to $30^{\circ}$ of extension deficit.

- Week 5: Full elbow flexion, up to $20^{\circ}$ of extension deficit.

- Week 6: Full elbow flexion, up to $10^{\circ}$ of extension deficit.

\section{Strengthening Program}

Single plane active ROM elbow flexion, extension, supination, and pronation.

\section{Week 7-11}

Full range of motion of the elbow; discontinue the brace if adequate motor control.

The patient may begin composite motions (i.e. extension with pronation).

If at 8 weeks post-operatively the patient has significant range of motion deficits, therapist may consider more aggressive management after consultation with referring surgeon.

\section{Strengthening Program}

A progressive active-resistance exercise program is initiated for elbow flexion, extension, supination, and pronation.

\section{Week 12}

Standard removal of the Hinged Elbow Brace.

The patient may initiate light upper extremity weight training.

\section{Strengthening Program}

Initiation of endurance program that simulates desired work activities/requirements.

Stimulation of elbow and shoulder range of motion, strength and coordination. 